\title{
VARIACIÓN DE COBERTURAS FORESTALES Y OCUPACIÓN DEL TERRITORIO EN EL MUNICIPIO DE ARMENIA 1939-1999
}

\author{
Olga Alicia Nieto C. ${ }^{1}$ \\ Luis Fernando Jiménez N. ${ }^{2}$ \\ Margarita Nieto R. ${ }^{3}$
}

Recibido el 5 de noviembre de 2014, aprobado el 2 de julio de 2015 y actualizado el 10 de noviembre de 2015

DOI: 10.17151/luaz.2016.42.19

\section{RESUMEN}

Introducción: Al observar la variación de la cobertura vegetal en un territorio, se puede observar su historia escrita sobre ese paisaje. Objetivo: El presente artículo pretende describir la variación de coberturas vegetales en relación con la dinámica de ocupación y poblamiento de la ciudad de Armenia, desde el año 1939 hasta el año 1999. Método: La serie de aerofotografías del municipio de Armenia existentes en el IGAC, desde 1939 hasta 1999, se georreferenciaron, se construyeron los mosaicos para cada uno de los años, se calcularon las áreas a través de una herramienta SIG (Sistema de Información Geográfica) y se determinaron coberturas vegetales y áreas pobladas. Se comparó la variación año a año con la dinámica de ocupación y el poblamiento del territorio. Resultados: El área urbanizada creció de 132,9 ha en 1939, a 1.316,23 ha en 1999. El área en coberturas vegetales creció de 82,52 ha que representaban el $38,32 \%$ del área poblada en 1939 , hasta 1.048 ha que representaban el $45,35 \%$ del perímetro urbano en 1999. Conclusión: El porcentaje de cobertura vegetal aumentó y ahora se considera suelo de protección, lo cual confiere especiales características al paisaje de Armenia, que debe ser protegido y conservado.

\section{PALABRAS CLAVE}

Cobertura vegetal, paisaje, territorio, SIG, gestión de servicios ambientales.

\section{VARIATION OF FOREST COVERAGE AND OCCUPATION OF THE LAND IN THE MUNICIPALITY OF ARMENIA 1939-1999 ABSTRACT}

Introduction: By observing the variation of the vegetation coverage in an area, it is possible to see its history written on the landscape. Objective: This article aims to describe the variation of vegetation coverage in relation to the dynamics of occupation and settlement of the city of Armenia, from 1939 until 1999. Method: The series of aerial photographs (by Agustin Codazzi Geographical Institute IGAC) of the city of Armenia from 1939 to 1999 were georeferenced and mosaics for each of the years were assembled by calculating the areas through a GIS tool. Vegetation coverage and urban land use were determined. The variation year to year was compared with the dynamics of occupation and settlement of the territory. Results: The urbanized area has increased from 132.9 hectares in 1939 to 1316.23 hectares in 1999 . The vegetation coverage area rose from 82,52 hectares which represented $38.32 \%$ of the populated area in 1939 , to 1,048 hectares, representing $4535 \%$ of the city limits in 1999. Conclusion: The percentage of vegetation coverage increased and 
is now considered protected land which gives special characteristics to the landscape of Armenia that must be protected and conserved.

KEY WORDS: Vegetation coverage, landscape, territory, GIS, environmental management services.

\section{INTRODUCCIÓN}

Según Morláns (2005), se puede definir el paisaje como: "Una parte de la superficie terrestre reconocible, que resulta y es mantenida por la mutua actividad de seres vivos y no vivos, incluyendo entre los primeros al hombre" (p. 4) o:

Una parte del espacio sobre la superficie terrestre, la cual consiste en un complejo de sistemas, formado por actividad de la roca, del agua, del aire de las plantas, de los animales y el hombre y por su fisonomía constituye una entidad reconocible. (p. 5).

En efecto, actualmente se afirma que cualquier fragmento de la superficie terrestre (fondos oceánicos incluidos), intervenido o no por los humanos, configura un paisaje; es decir, un conjunto de referentes físicos y funcionales, susceptible de ser considerado como un fenómeno real en sí mismo. El paisaje refleja la realidad ambiental de cada lugar (geológica, climática, edáfica), a la vez que resume y expresa la historia de procesos biológicos y antrópicos que se hayan podido desarrollar en él.

La interacción entre los factores formadores del paisajes y los elementos del paisaje, entre los que se suman el clima, los suelos, la litología, la vegetación, la fauna y las actividades humanas (Etter, 1991), diferencian un determinado paisaje frente a otros de relieves similares, a la vez que contribuyen a su transformación. El paisaje resulta ser el aspecto general de una región, configurado por la modelación de distintos factores (abióticos, bióticos y antrópicos) cuya particular historia evolutiva y adaptativa le confiere ciertas peculiaridades (Morláns, 2005).

De esta forma, el paisaje se entiende como aquel espacio resultante de la relación Naturaleza-Sociedad; así el análisis de estos espacios socionaturales, amerita una visión histórica, una dimensión espaciotemporal entendida desde su permanencia, evolución, transformación, cambio y desaparición de procesos abióticos y bióticos, como también de las modificaciones surgidas desde la creación del paisaje sociocultural (Molano, 1990).

La variación de las coberturas vegetales muestra cómo la configuración del paisaje es dinámica y expresa el resultado de la interacción entre los ecosistemas y las comunidades que los habitan, en especial las comunidades humanas que los transforman y los dibujan permanentemente. Dinámicas humanas como la expansión de la frontera agrícola, el cambio de los usos del suelo, el urbanismo, modifican e influyen constantemente en las relaciones ecológicas de los bosques, de los ríos y de los ecosistemas en general.

La información cartográfica digital actualizada y de alta resolución que permita conocer cambios multitemporales de cobertura vegetal y de uso del suelo es importante en la investigación de los recursos naturales. 
Teniendo en cuenta que la composición y estructura de las coberturas vegetales determinan intercambios de agua y energía en un paisaje, estudiar sus características serán insumos útiles para la identificación y descripción de dinámicas asociadas con servicios que para el bienestar humano se reconocen en un territorio, como es el ámbito urbano. Algunos de estos servicios, entendidos como las contribuciones directas e indirectas de los ecosistemas al bienestar humano (De Groot, 2013), pueden ser la provisión y regulación hídrica, la regulación del clima y del aire, la conservación de la biodiversidad, la mitigación de desastres naturales, el valor de existencia y la oportunidad de espacios verdes, etc.

Debido a la dinámica y variabilidad de los fenómenos ocurridos en el tiempo la información digital cartográfica disponible en el país pierde vigencia permanentemente (Rincón-Romero et al., 2012), sin embargo, su uso para el análisis de estos fenómenos es indispensable para la definición de soluciones informadas y estrategias pertinentes, que en el marco de un proceso de ordenamiento territorial estén enfocadas hacia el desarrollo sostenible, la conservación del medio ambiente, la mitigación de los impactos de los procesos naturales o productivos y en la adaptación o prevención ante los desastres naturales, entre otros.

La Fundación Semillas de Vida ha desarrollado un trabajo de caracterización y ha gestionado un proceso de conservación de las microcuencas hidrográficas urbanas de la ciudad de Armenia en el marco del ordenamiento territorial del municipio. En este proceso ha considerado importante hacer un análisis de la variación de coberturas vegetales, desde el año 1939 hasta el año 1999 y compararlo con la dinámica de ocupación y el poblamiento del territorio, con base en las series de aerofotografías del municipio de Armenia existentes en el IGAC, desde 1939 hasta 1999.

Se pretende que esta visión retrospectiva aporte elementos para ver el futuro del municipio de Armenia y que esta visión histórica de construcción y modificación del paisaje permita incorporar elementos para una planeación prospectiva de la ciudad en la cual las dinámicas de las coberturas vegetales, su estado, características y usos, aporten argumentos para el reconocimiento de su relevancia y relación con el bienestar y la calidad de vida de sus habitantes.

\section{Área de estudio}

Está ubicada en el área urbana del municipio de Armenia, departamento del Quindío, que se encuentra enmarcada dentro de las siguientes coordenadas con origen Bogotá, con un área según la base de datos del SIG de la Fundación Semillas de Vida de 2.361,8 hectáreas y 350.000 habitantes según el censo de 1993 (Fundación Semillas de Vida, 2005). 
Tabla 1. Coordenadas planas de la ubicación de la ciudad de Armenia (Quindío)

\begin{tabular}{|c|c|}
\hline Esquina Nor-Oeste: & $1^{\prime} 151.000 \mathrm{~m} . \mathrm{E}$. con $999.000 \mathrm{~m} . \mathrm{N}$. \\
\hline Esquina Nor-Este: & 1'159.500 m.E. con 999.000 m.N. \\
\hline Esquina Sur-Oeste: & $1 ' 151.000 \mathrm{~m} . \mathrm{E}$. con $989.500 \mathrm{~m} . \mathrm{N}$. \\
\hline Esquina Sur-Este: & $1^{\prime} 159.500 \mathrm{~m} . \mathrm{E}$ con $989.500 \mathrm{~m} . \mathrm{N}$ \\
\hline
\end{tabular}

Fuente: Base de datos SIG. Fundación Semilla de Vida (2005).

La zona urbana de Armenia está ubicada sobre el abanico volcánico del Quindío, limitada al occidente y oriente por los cañones de la quebrada Hojas Anchas y el río Quindío respectivamente, presenta altos valores de pendiente que condicionan la expansión urbana hacia el norte y el sur. La ciudad se concentra sobre una zona donde abundan cañadas que la atraviesan con una distancia promedio entre ellas de $200 \mathrm{~m}$ (Secretaría de Salud Pública de Armenia, 2000).

El municipio de Armenia (Quindío), tiene una especial connotación, por estar atravesado por 122 drenajes, de los cuales 54 son quebradas urbanas, que conforman 18 microcuencas o unidades de manejo (CRQ y Fundación Semillas de Vida, 2001), que constituyen una potencialidad como corredores de conservación urbanos (Figura 1). En estos corredores, longitudinales a las quebradas, se encuentra la mayor área en coberturas vegetales.

Estas áreas se ven cada día más expuestas a nuevos impactos ambientales, el más evidente es el actual proceso de construcción y expansión urbana. Con anterioridad se habían identificado impactos severos como: la pérdida de cobertura (67,42 ha entre 1996-2004), asentamientos humanos sobre suelo de protección, presencia de 2.416 viviendas en zona de riesgo, con un promedio de 4,2 habitantes por vivienda, es decir 10.220 personas aprox., contaminación hídrica por coliformes fecales con un rango mínimo de 2.300 NMP y máximo de 240.000.000 NMP de las quebradas de Armenia. Y como impactos moderados: la tala del bosque y guaduales, que ocasiona fragmentación de ecosistemas; la pérdida de hábitat que ofrece alimentación y vivienda a la fauna; la contaminación de aguas, nacimientos y quebradas, por la disposición de residuos sólidos y escombros (Salazar y Nieto, 2005). 


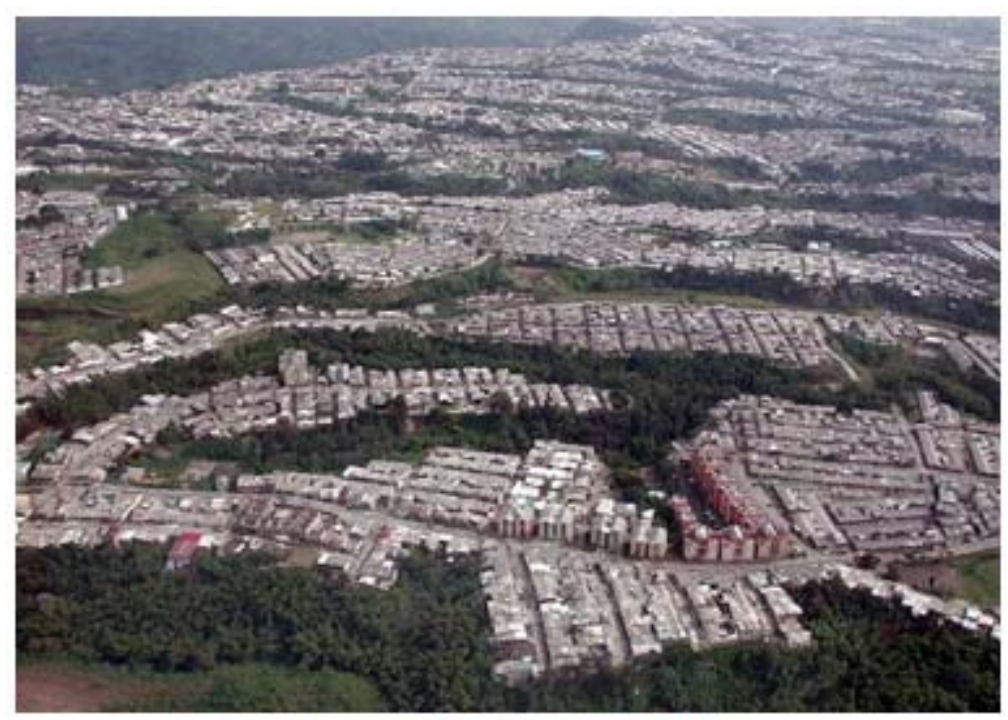

Fuente: Fundación Semillas de Vida.

Figura 1. Panorámica de Armenia.

En el marco del Plan de Ordenamiento Territorial, el municipio definió el suelo de protección que incluye ecosistemas estratégicos, zonas de fragilidad ecológica, fragmentos y relictos (Plan de Ordenamiento Territorial de Armenia, 1999). Buena parte de este suelo de protección se encuentra ubicado en los quiebres de pendiente, drenajes, rondas de las quebradas y zonas de riesgo por deslizamientos, inundaciones y sismos. Estas áreas, consideradas como estratégicas para la ciudad poseen un área total aproximada de 774,6 ha, de las cuales 358,6 se encuentran en fragmentos de bosque, 101,5 en agroecosistemas y 314,5 en recuperación ambiental (CRQ y Fundación Semillas de Vida, 2001). Estos suelos de protección, han sido considerados por el municipio como una potencialidad, razón por la cual constituyó el primer Sistema Municipal de Áreas Protegidas en el año 2000, considerándolos como un área de manejo especial urbano-rural [Decreto 140 de 2000 (Alcaldía de Armenia, 2000)].

En el año 2009 se adopta una revisión del Plan de Ordenamiento Territorial (2009-2023) que plantea los siguientes ejes estructurantes: la estructura ecológica principal, los bienes públicos y ciudadanía, la conectividad y redes, y el hábitat y desarrollo económico. En términos ambientales, sociales y económicos es una herramienta completa que define el ordenamiento del territorio basado en su sistema espacial, estructural y funcional interrelacionado, definido por un conjunto de elementos que garantizan el mantenimiento de la integridad de la biodiversidad y la provisión de servicios ecosistémicos para garantizar la satisfacción de las necesidades básicas para los habitantes y la articulación territorial como base del modelo de ocupación.

Sin embargo, actualmente el Sistema Municipal de Áreas Protegidas y las áreas que comprende, no fue homologado, ni recategorizado a una de las categorías vigentes, después de la reglamentación del Sistema Nacional de Áreas Protegidas (SINAP) y las categorías de áreas protegidas que lo conforman [Decreto 2372 de 2010 (Ministerio de Ambiente, Vivienda y Desarrollo Territorial, 2020)]. 


\section{MATERIALES Y MÉTODO}

\section{Definición de variables}

\section{Cobertura vegetal}

El concepto de "cobertura vegetal", se refiere al área que está cubierta por vegetación, en un territorio o área determinada:

[...] la cobertura vegetal puede ser definida como la capa de vegetación natural que cubre la superficie terrestre, comprendiendo una amplia gama de biomasas con diferentes características fisonómicas y ambientales, que van desde pastizales hasta las áreas cubiertas por bosques naturales. También se incluyen las coberturas vegetales inducidas que son el resultado de la acción humana, como serían las áreas de cultivos. (Rincón Romero et al., 2012, p. 2)

El área en cobertura vegetal usualmente se mide en porcentaje del área que es punto de referencia. Cuando se mide el porcentaje de cobertura vegetal sucesivamente en varios años, se puede hablar de variación de la cobertura en ese territorio. Entender estas variaciones puede apoyar la caracterización de algunas funciones y relaciones ecológicas, teniendo en cuenta las interacciones que tienen las coberturas vegetales con las dinámicas del agua y la energía en un ecosistema. En este sentido, es útil para la comprensión en términos de provisión de ciertos servicios ecosistémicos.

Según el IDEAM (1996), en su documento de coberturas vegetales, uso y ocupación del espacio, "la cobertura vegetal está definida como aquella unidad delimitada a partir de imágenes de satélite por sus características fisionómicas y ambientales" (p. 2). En Colombia se diferencian 40 unidades, agrupadas en tres categorías principales: boscosas, no boscosas y agroecosistemas.

Ecosistemas boscosos colombianos: Presenta las áreas boscosas del país identificadas y clasificadas en el mapa de coberturas vegetales, uso y ocupación del territorio. En Colombia se estima un área total de $63^{\prime} 777.519$ ha de coberturas boscosas (55,9\% del territorio). Escala 1:500.000 (IDEAM, 1996).

Ecosistemas no boscosos colombianos: Agrupa aquellos espacios naturales con vegetación achaparrada, de bajo porte, con uno a tres estratos que no superan los $3 \mathrm{~m}$ de altura (xerofitia andina, xerofitia basal, sabanas naturales, páramos, cobertura especial rupícola, insulares y sammofitia). Se estima un área total de 20 '671.960 ha $(18,1 \%$ del territorio). Escala 1:500.000 (IDEAM, 1996).

Agroecosistemas colombianos: Representan los espacios naturales modificados total o parcialmente por el hombre para el desarrollo de actividades agropecuarias en el país. Se calcula un área total de $29 ' 210.512$ ha (25,6\% de la superficie del país). Escala 1:500.000 (IDEAM, 1996). 
Para el caso específico de Armenia, el indicador de cobertura vegetal, retoma diferentes tipos de cobertura, según la información generada por el Fundación Semillas de Vida (Fundación Semillas de Vida, 2005; Salazar y Nieto, 2005) y se han agrupado estas coberturas en:

- Bosques: bosque húmedo pre montano, incluye bosque maduro, intermedio, temprano, guadual y café con sombrío.

- Cultivos: café, yuca, plátano, son los más evidentes.

- Pastizales: pasto sin ganadería.

- Potreros (incluye ganado).

Esta clasificación de coberturas vegetales puede hacerse equivalente a la definida por el IDEAM. Es sobre este indicador de cobertura vegetal, con las especificaciones dadas, que se lleva a cabo el presente análisis.

\section{Ordenamiento territorial}

De acuerdo a la Ley 388 de 1997, el ordenamiento territorial se define "como el conjunto de objetivos, directrices, políticas, estrategias, metas, programas, actuaciones y normas adoptadas para orientar y administrar el desarrollo físico del territorio [...]" y "[...] regular la utilización, transformación y ocupación del espacio, de acuerdo con las estrategias de desarrollo socioeconómico y en armonía con el medio ambiente y las tradiciones históricas y culturales" (Congreso de Colombia, 1997).

\section{Dinámicas de ocupación y poblamiento en relación con la base natural con bienestar y calidad de vida de los habitantes}

La sostenibilidad de las áreas urbanas y el bienestar de sus habitantes están soportados por la capacidad de los ecosistemas. Los resultados de las relaciones y funciones ecológicas sirven a la población directamente o indirectamente y pueden estar dentro o por fuera de la ciudad. La producción de comida, el abastecimiento de agua, la regulación hídrica, la regulación del aire, la conservación de la biodiversidad y el valor de existencia, son algunos de los servicios que pueden ser reconocidos y usados por los habitantes de una ciudad. Reconocer que esta capacidad es limitada y a su vez puede limitar factores sociales y económicos, debe tenerse en cuenta de manera explícita en la planificación del desarrollo de la ciudad (Folke et al., 1997).

Esta base y soporte natural es la que reconoce el POT del municipio de Armenia (2009-2023) como uno de sus ejes estructurantes, siendo la estructura ecológica soporte de la articulación territorial, base del modelo de ocupación y proveedor de las necesidades básicas para los habitantes.

\section{Identificación de las áreas en cobertura forestal, en cada una de las aerofotografías}

Se consiguieron las aerofotografías de los años 1939, 1946, 1954, 1967, 1973, 1984 y 1999, con el IGAC (1939, 1946, 1954, 1967, 1973, 1984, 1999).

Se identificaron más de 200 puntos clave en la ciudad, que fueron puntos de referencia en las fotos para la herramienta SIG (Fundación Semillas de Vida, 2005). Se construyeron los mosaicos con las fotos de los sobrevuelos, por cada uno de los años mencionados. Se determinaron las coberturas en cada una de las aerofotografías, discriminando coberturas 
vegetales de áreas pobladas. Y se discriminaron las coberturas vegetales en: bosques, pastizales, cultivos, y potreros. En las áreas pobladas se incluyeron construcciones, vías y lotes. A través del Sistema de Información Geográfica (SIG) se calcularon las áreas.

\section{Comparación de las áreas con el área poblada y con el perímetro} urbano existente a la fecha

Se hizo una comparación entre áreas con coberturas vegetales y perímetro del año 2004, especialmente en cuanto a los suelos de protección definidos por el POT en 1999.

Se compararon las coberturas vegetales para cada uno de los años mencionados, con el área poblada que se evidencia en las aerofotografías y se analizó la cobertura vegetal con referencia a la dinámica de poblamiento del municipio, pero como no se contaba con los perímetros urbanos de todos los años, se delimitó el área poblada, que involucraba el área urbana y las coberturas adyacentes.

\section{Análisis de la variación de las coberturas vegetales en el municipio de Armenia}

Se estableció una tabla que relacionó áreas pobladas y áreas en coberturas año a año y se identificó el porcentaje de cobertura respecto a cada área en esos años.

Se comparó la variación año a año. Y se describió luego en relación con hechos históricos relevantes en el poblamiento del municipio y se relacionaron con la variación de coberturas vegetales, para el municipio en esos años.

Se construyeron mosaicos con las aerofotografías de los años enunciados y se analizaron las coberturas vegetales respecto al área poblada (área urbanizada más área en cobertura vegetal) y/o al perímetro urbano existente.

\section{RESULTADOS}

En los 60 años del período analizado (Tabla 2), el área urbanizada creció de 132,9 ha en 1939 a 1.316,23 ha en 1999.

El área en coberturas vegetales creció de 82,52 ha que representaban el $38,32 \%$ del área poblada en 1939 , hasta 1.048 ha que representaban el 45,35\% del perímetro urbano en 1999. 
Tabla 2. Variación de coberturas vegetales (ha y \%)

\begin{tabular}{|l|c|c|c|c|c|c|c|}
\hline & \multicolumn{6}{|c|}{ VARIACION DE COBERTURAS 1939-1999 (ha) } \\
\hline DESCRIPCION & 1939 & 1946 & 1954 & 1967 & 1973 & 1984 & 1999 \\
\hline AREA POBLADA & 215,46 & 350,53 & 542,41 & 1043,42 & 1186 & 1542,69 & \\
\hline $\begin{array}{l}\text { AREA } \\
\text { URBANIZADA }\end{array}$ & 132,9 & 219,97 & 356,17 & 537,56 & 651,52 & 843,25 & 1316,23 \\
\hline Porcentaje (\%) & $61,68 \%$ & $62,75 \%$ & $65,66 \%$ & $51,51 \%$ & $54,93 \%$ & $54,66 \%$ & $55,65 \%$ \\
\hline $\begin{array}{l}\text { COBERTURAS } \\
\text { VEGETALES } \\
\text { Porcentaje (\%) }\end{array}$ & 82,56 & 130,74 & 186,23 & 505,92 & 534,48 & 699,44 & 1048,72 \\
\cline { 2 - 8 } & $38,32 \%$ & $37,25 \%$ & $34,34 \%$ & $48,49 \%$ & $45,07 \%$ & $45,34 \%$ & $45,35 \%$ \\
\hline BOSQUES & 24,48 & 37,76 & 86,99 & 358,87 & 260,12 & 440,82 & 456,12 \\
\hline Porcentaje (\%) & $11,36 \%$ & $10,77 \%$ & $16,03 \%$ & $34,39 \%$ & $21,93 \%$ & $28,57 \%$ & $19,28 \%$ \\
\hline CULTIVOS & & & & 16,90 & & 12,41 & 92,99 \\
\hline Porcentaje (\%) & & & & $1,61 \%$ & & $0,80 \%$ & $3,93 \%$ \\
\hline PASTIZALES & 12,80 & 21,41 & 21,51 & 50,13 & 132,72 & 112,53 & 308,36 \\
\hline Porcentaje (\%) & $5,94 \%$ & $6,1 \%$ & $3,96 \%$ & $4,8 \%$ & $11,19 \%$ & $7,29 \%$ & $13,03 \%$ \\
\hline POTREROS & 45,28 & 71,57 & 77,73 & 80,02 & 141,64 & 133,68 & 191,25 \\
\hline Porcentaje (\%) & $21,01 \%$ & $20,41 \%$ & $14,33 \%$ & $7,66 \%$ & $11,94 \%$ & $8,66 \%$ & $8,08 \%$ \\
\hline PERIMETRO & & & & & & & 2364,9 \\
\hline
\end{tabular}

Fuente: Fundación Semillas de Vida.

El aumento de coberturas vegetales en área y en porcentaje se debe a que inicialmente el área urbana se estableció en la parte plana, pero paulatinamente se fueron urbanizando las partes altas de las ondulaciones de las microcuencas, y se involucraron las quebradas con sus bosques al área poblada y al perímetro urbano del municipio (Figura 2).

Sobresalen los años de 1967, 1973 y 1984, como los años en los cuales hay mayor porcentaje de cobertura vegetal: $48,49 \%$ a $45,34 \%$ (Tabla 2), que coinciden con la época en la cual la expansión del poblamiento del municipio se dio hacia el Norte y el Sur. 


\section{CRECIMIENTO URBANO DEL MUNICIPIO DE ARMENIA UNARETROSPECTIVA 1939 - 1999}
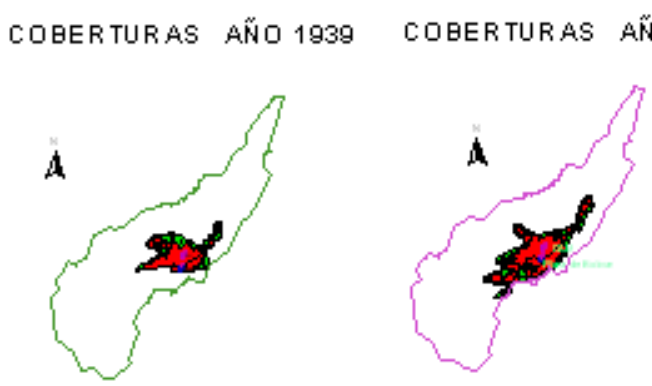

COBERTURAS AÑ̃ 1954

\section{COBERTURAS AÑN 1967}
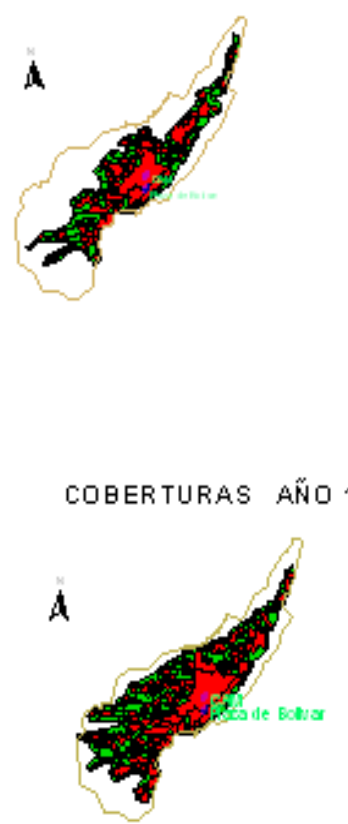

\begin{abstract}
COBERTURAS ANNO 1973
\end{abstract}
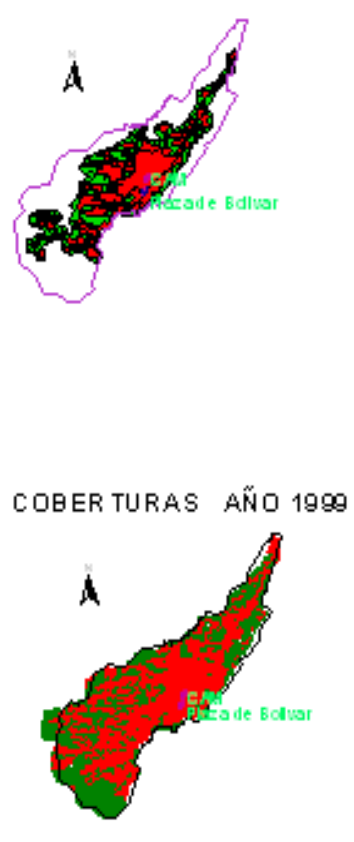

Figura 2. Variación del perímetro municipal, del área urbanizada (en rojo) y de las áreas con cobertura vegetal (en verde).

En el año 1939 (Figura 3) el área poblada era de 215,46 ha, de las cuales el $61,68 \%$, es decir 132,9 ha, constituían el área urbanizada y el $38,32 \%$ (82,56 ha) el área en cobertura vegetal, predominando el área en potreros con 45,28 ha y el área en bosques con 24,48 ha. 


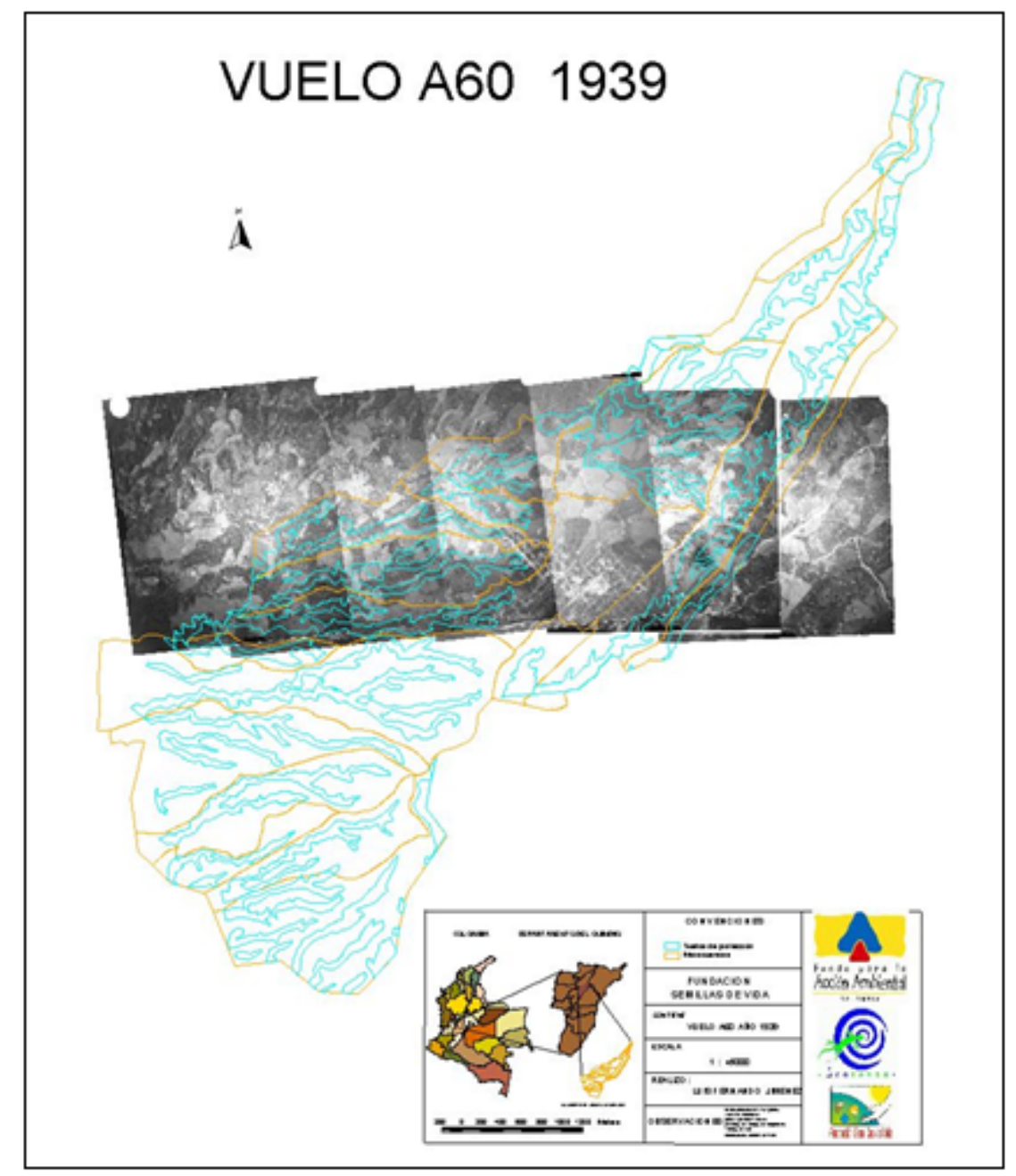

Figura 3. Area poblada y área con cobertura vegetal. Sobrevuelo 1939.

En el año 1946 (Figura 4) el área poblada era de 350,53 ha, de las cuales el 62,75\%, es decir 219,79 ha, constituían el área urbanizada y el 37,32\% (130,74 ha) el área en cobertura vegetal, predominando en ésta el área en potreros con un 71,57 ha, seguida por el área en bosques con 37,76 ha.

Sobre el uso del suelo, la expansión del área poblada es principalmente urbanización y en el área en coberturas predomina la ganadería.

En la aerofotografía, la cuadrícula de la ciudad evidencia en el Sur el puente de Don Nicolás, la estación del ferrocarril y el cementerio (ahora terminal de transporte), en el Norte se identifica el Parque Fundadores y la Nueva Cecilia. 


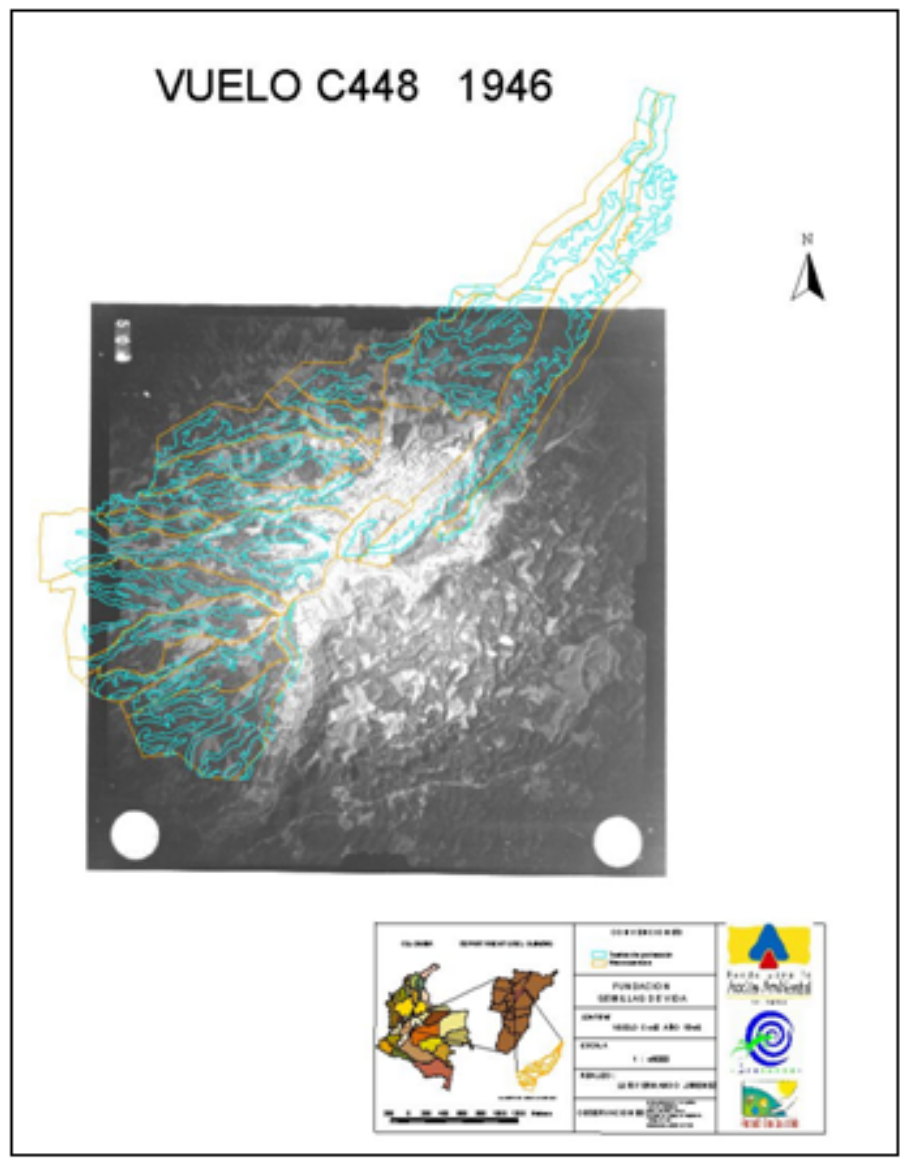

Figura 4. Area poblada y área con cobertura vegetal. Sobrevuelo 1946.

En el año 1954 (Figura 5) el área poblada era de 542,41 ha, de las cuales el $65,66 \%$, es decir 356,18 ha, constituían el área urbanizada y el $34,34 \%$ $(186,23 \mathrm{ha})$ el área en cobertura vegetal, con un incremento en el área en bosques con 86,99 ha, seguida por el área en potreros con 77,73 ha.

En la aerofotografía, la cuadrícula de la ciudad crece en sentido CentroOccidental, hacia barrios como El Granada. 


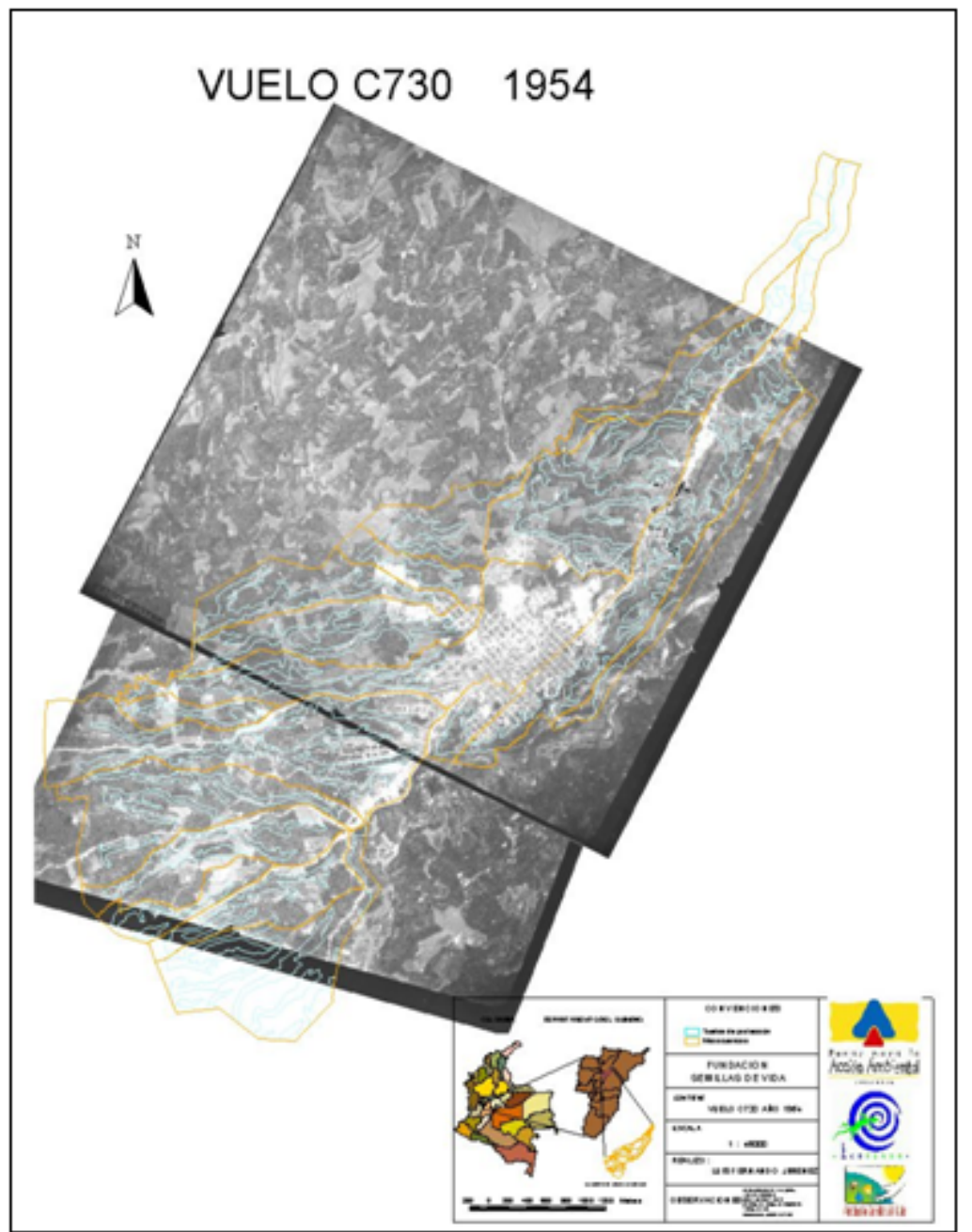

Figura 5. Área poblada y área con cobertura vegetal. Sobrevuelo 1954

En el año 1967 (Figura 6) el área poblada era de 1.043,48 ha, de las cuales el 51,51\%, es decir 537,56 ha, constituían el área urbanizada y el $48,49 \%$ (505,92 ha) el área en cobertura vegetal, con un incremento en el área en bosques con 358,87 ha, seguida por el área en potreros con 80,02 ha.

En la aerofotografía, la cuadrícula de la ciudad se engrosa a lado y lado de la Carrera 14 y aparecen nuevas áreas urbanizadas en el Norte como la Universidad del Quindío, el Hospital y el barrio Nogal. 


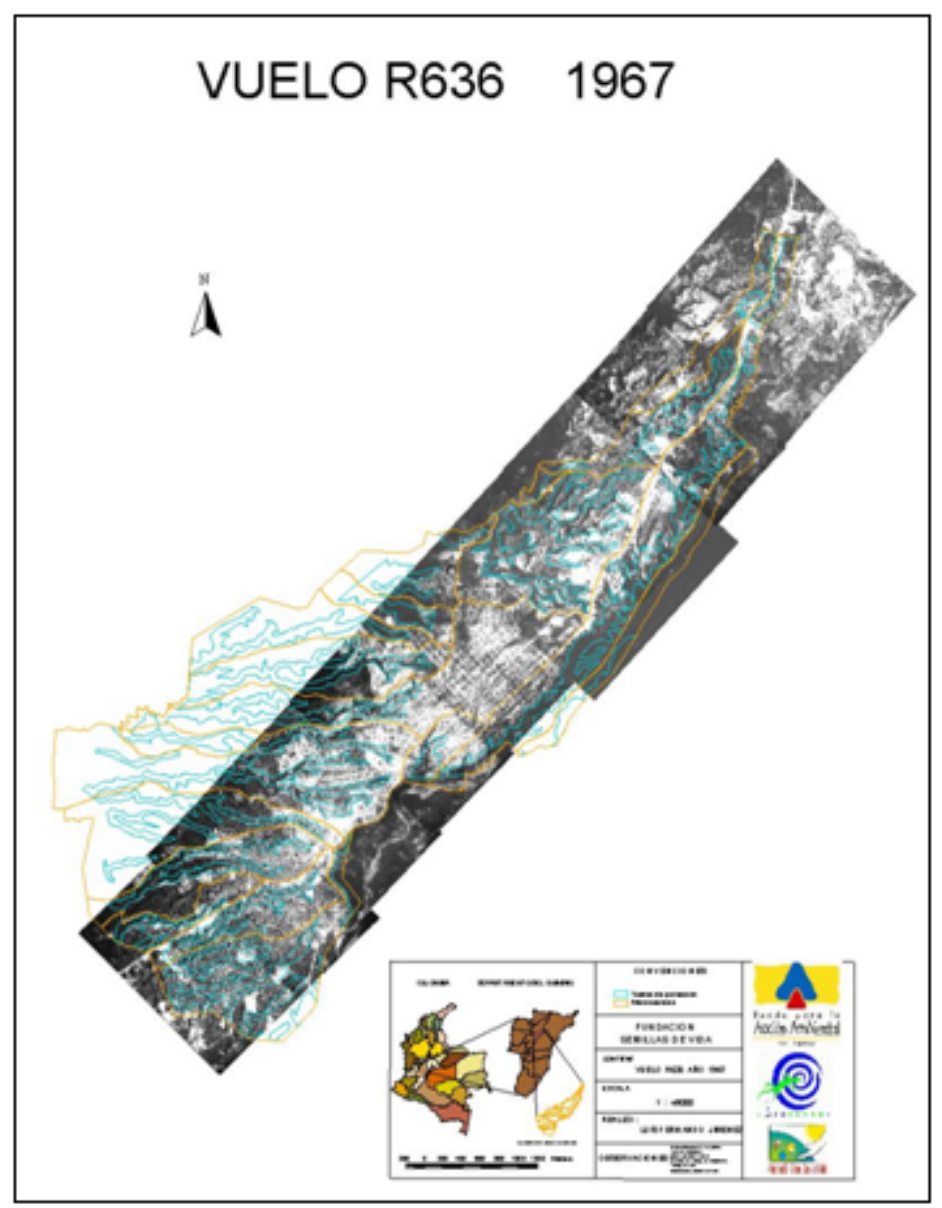

Figura 6. Área poblada y área con cobertura vegetal. Sobrevuelo 1967.

En el año 1973 (Figura 7) el área poblada era de 1.186 ha, de las cuales el 54,93\%, es decir 651,52 ha, constituían el área urbanizada y el $45,07 \%$ (534,48 ha) el área en cobertura vegetal, predominando el área en bosques con 260,12 ha, seguida por el área en potreros con 141,64 ha. Aumenta el área en pastizales a 132,72 ha.

En la aerofotografía, la cuadrícula de la ciudad crece en sentido SurOccidental. 


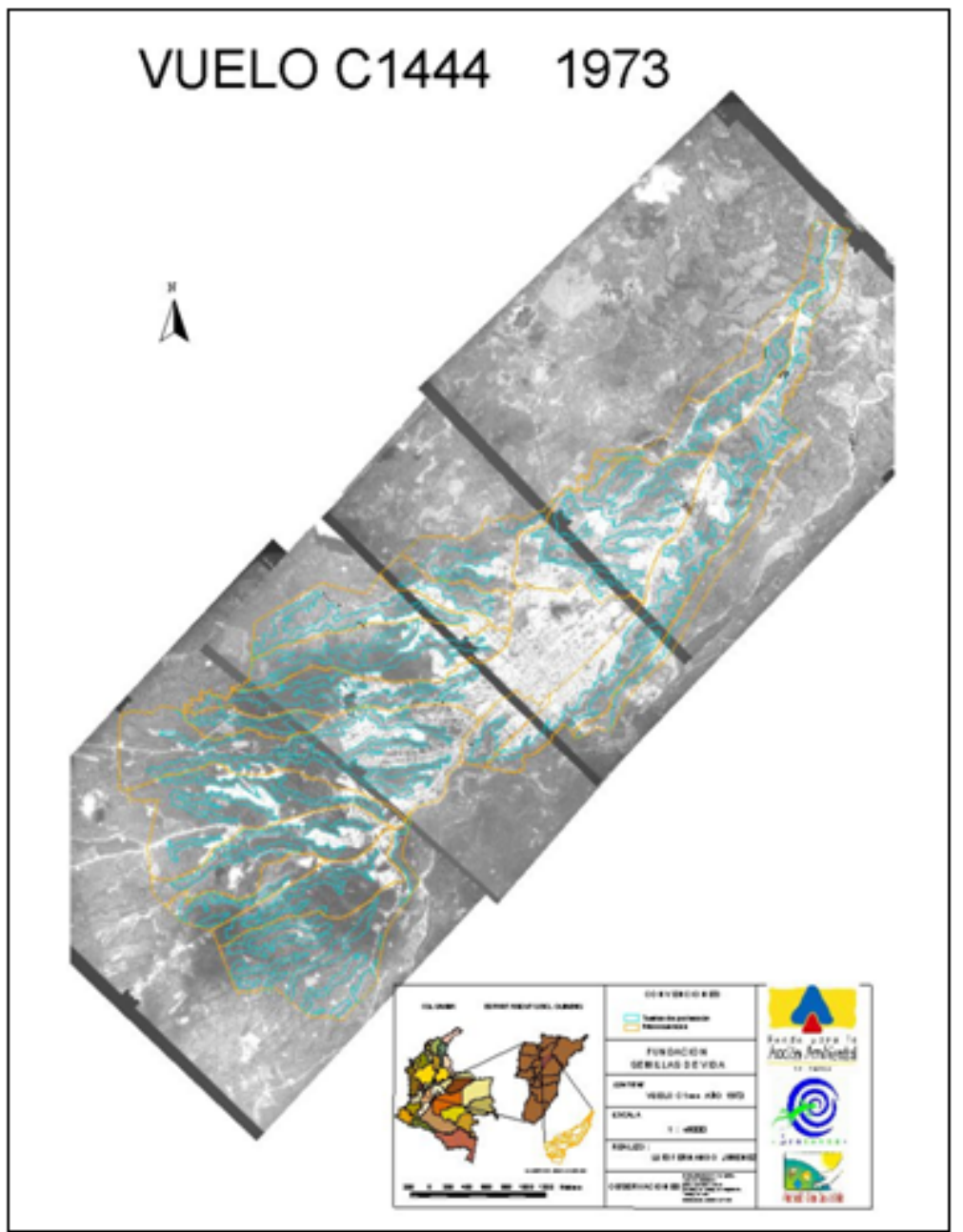

Figura 7. Area poblada y área con cobertura vegetal. Sobrevuelo 1973.

En el año 1984 (Figura 8) el área poblada era de 1.542,69 ha, de las cuales el $54,66 \%$, es decir 843,25 ha, constituían el área urbanizada y el $45,34 \%(699,44 \mathrm{ha})$ el área en cobertura vegetal, predominando el área en bosques con 440,82 ha, seguida por el área en potreros con 133,68 ha.

Disminuye el área de pastizales a 112,53 ha y reaparecen las áreas de cultivos con 12,71 ha. Demostrando que las zonas de barbecho fluctúan entre pasto y cultivos.

En la aerofotografía, la cuadrícula muestra crecimiento Sur-Occidental, al tiempo que el alargamiento de la ciudad en sentido Norte y Sur es evidente. 


\section{VUELO C2141 1984}

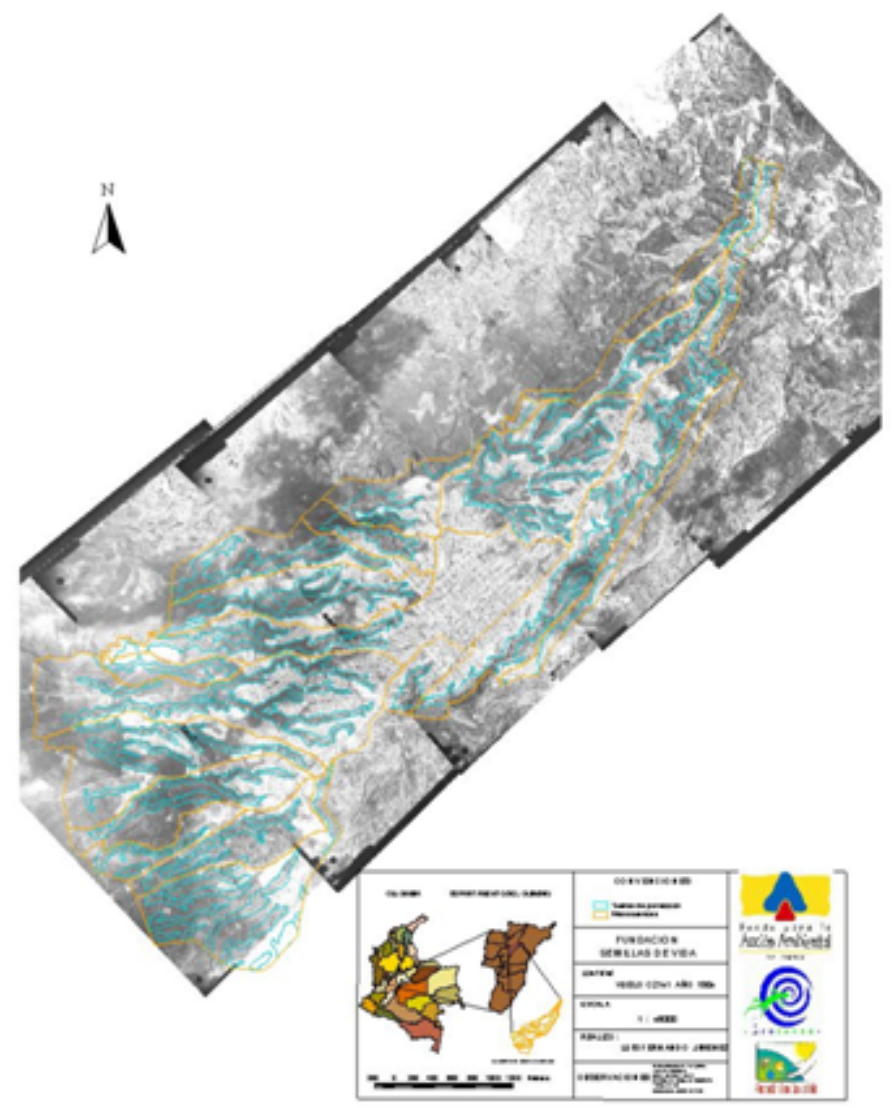

Figura 8. Área poblada y área con cobertura vegetal. Sobrevuelo 1984

En el año 1999 (Figura 9) el área dentro del perímetro urbano era de $2.365,29$ ha, de las cuales el 55,65\%, es decir 1.316 ha, constituían el área urbanizada y el $45,35 \%$ (1.048,72 ha) el área en cobertura vegetal, predominando el área en bosques con 456,12 ha, seguida por el área en pastizales con 308,36 ha.

Con respecto a 1984, aumenta el área de pastizales, disminuye el área de potreros (191,25 ha) y aumenta el área en cultivos (92,99 ha). En la aerofotografía, se superpone al actual perímetro de la ciudad y se observa cómo se incorporan las microcuencas (cañadas y drenajes), que es el área donde se encuentra la mayor área en bosques y otras coberturas vegetales. Para este año ya se había formulado el Plan de Ordenamiento Territorial que constituyó estas áreas en suelo de protección. 


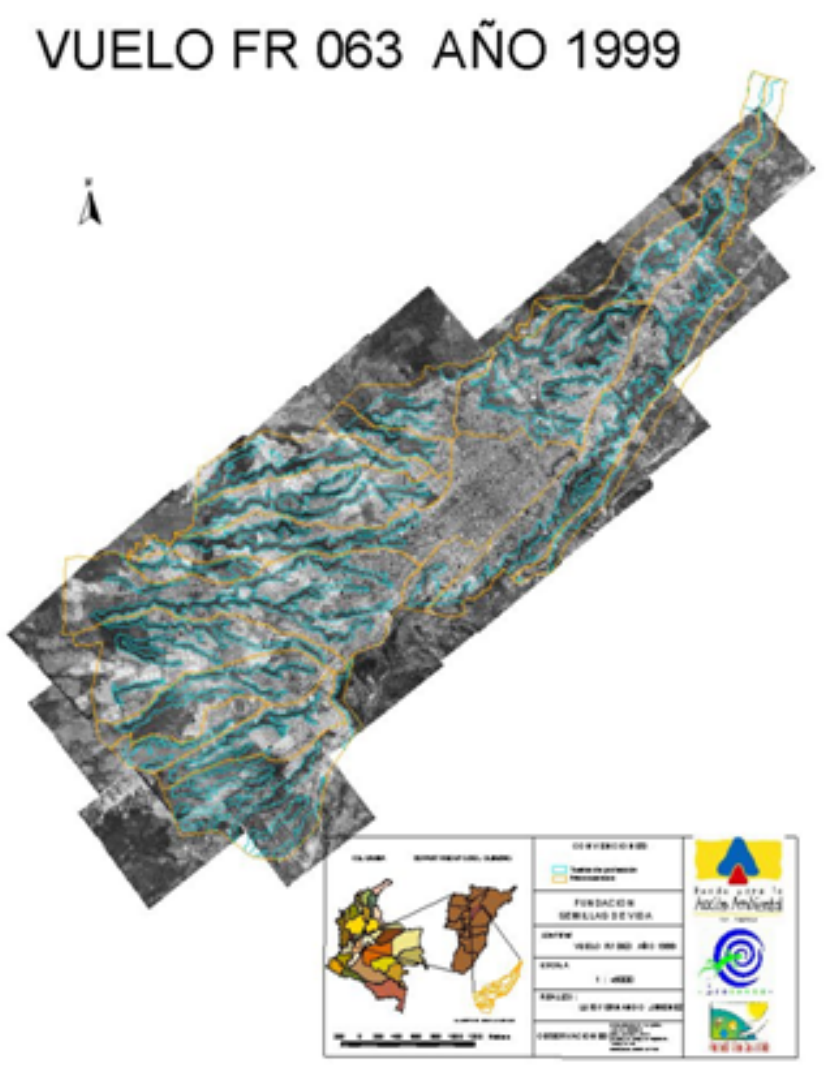

Figura 9. Área poblada y área con cobertura vegetal. Sobrevuelo 1999.

\section{DISCUSIÓN}

La variación de coberturas vegetales en el municipio de Armenia está ligada estrechamente a factores biofísicos como la geología, la topografía, el relieve y factores sociales como la dinámica de poblamiento y ocupación del territorio, los cuales definen el uso del suelo. Y en esta historia han sido dinamizadores de ambos, la fundación de la ciudad para tener un punto de abastecimiento antes de atravesar el río Quindío; el transporte primero en mula, luego férreo y después vial; la ganadería y la caficultura; la prestación de servicios de salud y educación; la política; el desastre y el proceso de reconstrucción. Más recientemente el turismo y en la actualidad el auge de la construcción.

\section{Crecimiento urbano en la ciudad de Armenia, una retrospectiva}

El municipio de Armenia fue fundado en 14 de octubre de 1889 , y en palabras de Miguel Ángel Rojas:

El caserío en bahareque y tierra era una cuadrícula que se expandía entre el bosque a golpe de hacha y machete. Armenia se empinó por la calle de Encima en un perfil coherente, y creció en la calle Real que se extendió por la calle de Sevilla y bajó hasta el puente de Don Nicolás a reivindicar el paso por el río Quindío, aquel que obligó a Jesús María Ocampo -El Tigrero- a fundar la ciudad. En los lotes desbrozados, arrancados a la selva, crecieron el maíz, la caña, el fríjol, el plátano y la yuca y fue abriéndose paso 
el potrero que con rapidez convirtió al Quindío de sus primeros años en un emporio ganadero y exportador de pieles. Al poco tiempo llegó el café a democratizar la hacienda oriental del siglo $\mathrm{XIX}$, cada colono sembró su pedazo de tierra hasta que el café empezó a inundar no sólo las laderas, sino los propios trapiches de cañas, los potreros, los maizales y hasta los patios de la casa. Armenia creció a un ritmo sorprendente, tanto que entre 1912 y 1930 tuvo un aumento poblacional de 548,6\%. En 1912 tenía 6.651 habitantes y en 1930 alcanzaba 36.494, razón por la cual el poeta Guillermo León Valencia, en la celebración de su cuadragésimo aniversario, dijo: "este es un milagro de ciudad". (Rojas, 1999, p. 47).

Éste era el paisaje que predominaba cuando en 1939, 50 años después de su fundación, se ubica en el punto de partida esta visión retrospectiva en aerofotografías.

A pesar de que el área en cultivos aparece como cero ha, es posible que ya el café con sombrío empezara a hacer parte de la cobertura conocida como "bosques".

La ciudad creció alrededor de la cuadrícula central hasta los años cincuenta. Y la economía se consolidó con la primera bonanza cafetera de 1957, que le mereció el título de capital cafetera de Colombia. Sin embargo, el crecimiento hacia el Sur tenía entonces puntos de referencia importantes como la estación del ferrocarril y las casas trilladoras de café que fueron concentrando sus negocios cerca al sitio de transporte. Otro punto de referencia era el cementerio, que por entonces se hallaba ubicado en el actual Terminal de pasajeros.

En 1946 el potrero superó los bosques, posiblemente a expensas de bosques y de café con sombrío (71,57 ha y 37,76 ha, respectivamente).

A ritmo de bonanza, entre 1960 a 1980, la ciudad empieza su expansión hacia el Norte con polos de desarrollo como la Universidad del Quindío y el Hospital, los cuales jalonan el proceso de urbanismo. Y empieza a crecer longitudinalmente la ciudad, por encima de sus quebradas y drenajes.

Posiblemente el área en bosque aumenta a expensas de bosques y de café con sombrío y se evidencia la disminución del área en ganadería.

Se pueden citar como periodos importantes los siguientes: Ascenso industrial hasta 1950, Descenso industrial en la época de 1980, Bonanzas cafeteras de 1976-77 y 1984-85 (Rojas, 1999).

En los 80 y los 90 la ciudad avanza en sentido Sur-Occidental construyéndose barrios por proselitismo político, tales como La Adiela y La Patria, entre otros.

Para 1999, Armenia contaba con más de 300.000 habitantes y el 97,5\% de su población habitaban las más de 220 urbanizaciones existentes.

No es posible ser ajenos a cómo el terremoto y el proceso de reconstrucción impulsaron el desarrollo urbanístico hacia el Sur-Oriente sobrepasando el actual Cementerio Jardines, el perímetro urbano y el perímetro de servicios, con urbanizaciones como Génesis, Bambusa, 
Nuestra Señora de la Paz, Guaduales del Edén, Simón Bolívar y Cañas Gordas, entre otras.

El impacto del terremoto afectó toda la estructura arquitectónica en la ciudad, lo cual no solo se atribuye a la fuerza de la naturaleza sino también a una serie de factores relacionados con la planeación que orientó la edificación de la ciudad. Cabe resaltar la reconstrucción de lo público: el CAM, el palacio de gobierno departamental, el cuartel de la policía, el batallón Cisneros, el aeropuerto, la ciudadela de la salud, el centro de cultura y la Universidad del Quindío. Se restauraron vías y avenidas cambiando totalmente la arquitectura de la ciudad.

Los años 90 también presencian la transformación de los barrios en los conjuntos cerrados, a raíz de la inseguridad y la violencia.

\section{CONCLUSIONES}

Si esta retrospectiva permite aproximarnos a los factores que han impulsado la dinámica de poblamiento y ocupación del territorio, también permite ver comparativamente cómo la cobertura vegetal ha cambiado ligada al uso del suelo que culturalmente predomine. Sin embargo, es esa cobertura la que aporta la sensación de verde que enamora a propios y visitantes, la que regula el clima, la que capta el agua de esas microcuencas, que da cobijo, madera y leña, y es el sitio donde se albergan más de 400 especies de flora y más de 70 de aves (Fundación Semillas de Vida, 2009).

Esta visión retrospectiva aporta elementos para que la gestión ambiental de la ciudad (Vásquez y Navarrete, 2014) dé prioridad a una relación armónica entre la cobertura vegetal y el proceso de urbanismo, entre el cemento y la calidad de vida y apunte realmente a construir una ciudadpaisaje en el contexto del Paisaje Cultural Cafetero recientemente reconocido por la UNESCO (Ministerio de Cultura de Colombia, 2011).

\section{AGRADECIMIENTOS}

Especial reconocimiento merece el Dr. Alfonso López Reina, geólogo e historiador, quien nos motivó a revisar la historia de este hermoso terruño en el cual vivimos y quien al conocer sobre la adquisición de las aerofotografías nos instó a hacerlo.

Al Proyecto Manejo Integral de microcuencas y áreas protegidas urbanas del Municipio de Armenia, que fue financiado por el Fondo para la Acción Ambiental a la Fundación Semillas de Vida, que facilitó la información y las aerofotografías.

\section{POTENCIAL CONFLICTO DE INTERESES}

No existe conflicto de intereses. 


\section{FUENTES DE FINANCIACIÓN}

No se tuvo una fuente de financiación.

\section{REFERENCIAS}

- Alcaldía de Armenia. (2000). Decreto 140 de 2000. Por el cual se crea el Sistema de Áreas Protegidas. Armenia: Autor.

- Alcaldía de Armenia. (1999). Plan de Ordenamiento Territorial del Armenia.

- Alcaldía de Armenia. (2009). Plan de Ordenamiento Territorial de Armenia 2009-2023.

- Arango, M., Branch, J.W. y Botero, V. (2005). Clasificación no supervisada de coberturas vegetales sobre imágenes digitales de sensores remotos: Landsat - ETM+. Revista Facultad Nacional de Agronomía, 58(1), 2611-2634.

- Congreso de Colombia. (1997). Ley 388 de 1997. Por la cual se armonizan y actualizan las disposiciones contenidas en la Ley $9^{\mathrm{a}}$ de 1989 con las nuevas normas establecidas en la Constitución Política, la Ley Orgánica del Plan de Desarrollo, la Ley Orgánica de Áreas Metropolitanas y la Ley por la que se crea el Sistema Nacional Ambiental.

- CRQ y Fundación Semillas de Vida. (2001). Formulación de criterios para la zonificación y manejo integral de los drenajes urbanos de Armenia. Armenia, Quindío: Autor.

- De Groot, R. (2013). Integrated Valuation of Biodiversity and Ecosystem Services for better Decision Making. Ponencia en el Simposio de Servicios Ecosistémicos. Bogotá, D.C.: Instituto de Investigación en Recursos Biológicos Alexander von Humboldt Universidad de los Andes.

- Etter, A. (1991). Introducción a la Ecología del Paisaje. Un marco de integración para los levantamientos rurales. Bogotá. Instituto Geográfico Agustín Codazzi, Subdirección de Docencia e Investigación, Unidad de Levantamientos Rurales. 1-34.

- Folke, C., Jansson, A., Larsson, J. y Constanza, R. (1997). Ecosystem Appropriation by Cities. Ambio, 26(3), 167-17.

- Fundación Semillas de Vida. (2009). Las aves del corredor de conservación urbano. Armenia: Autor.

- IDEAM. (1996). Mapa de coberturas vegetales, uso y ocupación del territorio. Memoria técnica. Bogotá: Autor. 
- IGAC. (1939, 1946, 1954, 1967, 1973, 1984, 1999). Aerofotografías de Armenia, 1939, 1946, 1954, 1967, 1973, 1984, 1999. Bogotá: Autor.

- Ministerio de Ambiente, Vivienda y Desarrollo Territorial. (2010). Decreto 2372 de 2010. Por el cual se reglamenta el Sistema Nacional de Áreas Protegidas. Ministerio de Ambiente, Vivienda y Desarrollo Territorial. Bogotá: Autor.

- Ministerio de Cultura de Colombia - Dirección de Patrimonio. (2011). Paisaje cultural cafetero. Un paisaje cultural productivo en permanente desarrollo. Bogotá: Autor.

- Molano, J. (1990). Villa de Leiva. Ensayo de interpretación social de una catástrofe ecológica. Fondo FEN Colombia.

- Morláns, M.C. (2005). El concepto de paisaje. Introducción a la Ecología del Paisaje. Catamarca: Editorial Científica Universitaria, Universidad Nacional de Catamarca.

- Rincón-Romero, M. Jarvis, A. y Mulligan, M. (2012).Cobertura vegetal en Colombia. Renata, 6 (4), 12-26.

- Rojas, M.A. Quiceno, O. (1999). Armenia, ayer y hoy. Armenia: Editorial Fudesco. Comité de Cafeteros del Quindío.

- Salazar, A. y Nieto, O.A. (2005). Plan de ordenamiento y manejo integral de microcuencas urbanas y áreas protegidas urbanas del municipio de Armenia. Armenia: Fundación Semillas de Vida.

- Secretaría de Salud Pública de Armenia (2000). Plan Malla Ambiental. Grupo Verde Ltda. Armenia: Autor.

- Fundación Semillas de Vida. (2005). Proyecto Manejo Integral de microcuencas y áreas protegidas del Municipio de Armenia, Quindío.

- Vásquez, D.M. y Navarrete, M. (2014). La gobernabilidad local en el contexto de la nueva gestión pública: requisito para la gestión ambiental urbana. Revista Luna Azul, 39, 63-88. Recuperado de http://lunazul.ucaldas.edu.co/index.php?option=content\&task=vie w\&id $=948$

1. PhD en Ciencias Biomédicas. Docente de Salud Pública, Programa de Medicina, Facultad de Ciencias de la Salud, Universidad del Quindío. Fundación Semillas de Vida. Armenia, Colombia. olgalicianieto@gmail.com

2. Magíster en Educación. Programa de Administración de Negocios, Facultad de Ciencias Económicas y Administrativas, Universidad del Quindío. Fundación Semillas de Vida. Armenia, Colombia. lufejini@gmail.com

3. Máster en Estudios Ambientales, Economía Ecológica. Instituto de Investigación en Recursos Biológicos Alexander von Humboldt. 
Integrante Fundación Semillas de Vida. Bogotá, Colombia. mnieto@humboldt.org.co

Para citar este artículo: Nieto C., O.A., Jiménez N., L.F. y Nieto R., M. (2016). Variación de coberturas forestales y ocupación del territorio en el municipio de Armenia 1939-1999. Revista Luna Azul, 42, 319-340. Recuperado de http://200.21.104.25/lunazul/index.php?option=com_content\&view=article\& id $=141$ 